\title{
Interphalangeal Joint 1
}

National Cancer Institute

\section{Source}

National Cancer Institute. Interphalangeal/oint 1. NCI Thesaurus. Code C102301.

A ginglymoid (hinge) synovial joint within the first digit of the hand or foot connecting the proximal and distal phalanges. 